\title{
Ferromagnetic nanoparticles with strong surface anisotropy: Spin structures and magnetization processes
}

\author{
L. Berger, Y. Labaye, and M. Tamine \\ Laboratoire de Physique de l'Etat Condensé, CNRS UMR 6087, Université du Maine, 72085 Le Mans, Cedex 9, France \\ J. M. D. Coey \\ School of Physics and CRANN, Trinity College, Dublin 2, Ireland
}

(Received 7 November 2007; published 24 March 2008)

\begin{abstract}
Monte Carlo simulations are used to investigate the effect of surface anisotropy on the spin configurations and hysteresis loops of ferromagnetic nanoparticles. Spherical particles of radius $\rho a$ are composed of $N$ atoms located on a simple cubic lattice with interatomic spacing $a$. The particles have $2 \leqslant \rho \leqslant 13$. A classical Heisenberg model is assumed, with surface and bulk anisotropy. When surface anisotropy is positive there are two types of ground states separated by a large energy barrier: a "throttled" configuration with reduced magnetization for intermediate values of surface anisotropy and a "hedgehog" configuration with zero magnetization in the strong surface anisotropy limit. Beyond a threshold, surface anisotropy of either sign induces $\langle 111\rangle$ easy axes for the net magnetization. Easy-axis hysteresis loops are then square, with a continuous approach to saturation, and the effective anisotropy is deduced either from the switching field or from the initial slope of the perpendicular magnetization curve. The hedgehog state shows a stepwise magnetization curve involving $\rho$ discrete configurations, and it passes to a throttled configuration before saturating. The hysteresis loop has the unusual feature that it involves a state in the first quadrant, which lies on the reversible initial magnetization curve; it is possible to recover the zero-field cooled state after saturation. A survey of the exchange and anisotropy parameters for a range of ferromagnetic materials indicates that the effects of surface anisotropy on the spin configuration should be most evident in nanoparticles of ferromagnetic actinide compounds such as US, and rare-earth metals and alloys with Curie points below room temperature; the effects in nanoparticles of $3 d$ ferromagnets and their alloys are usually insignificant, with the possible exception of FePt.
\end{abstract}

DOI: 10.1103/PhysRevB.77.104431

PACS number(s): 75.70.Rf, 75.30.Gw, 05.10.Ln, 75.40.Mg

\section{INTRODUCTION}

Magnetic nanoparticles are of interest both for fundamental reasons, and on account of their uses as ferrofluids, catalysts, and magnetic storage media. The magnetic properties of fine particles are influenced by surface effects, which become increasingly important as the particle size decreases. The surface effects are related to the broken symmetry of the crystal structure, whereas finite-size effects are directly attributed to the nanometric dimensions of the particles.

Much attention has been devoted to systems consisting of isolated nanoparticles for which experiments have indicated different magnetic properties of the surface and the bulk. ${ }^{1-8}$ The principal effects observed in ferromagnetic fine particles include the following.

(1) Ill-defined Curie temperature, which may be reduced compared to the bulk value.

(2) Decreased (sometimes increased) saturation magnetization $M_{s}$.

(3) Slow approach to magnetic saturation associated with surface spin disorder.

(4) Superparamagnetism.

(5) Single-domain behavior. The critical single-domain radius $R_{\mathrm{sd}}=36 \kappa l_{\mathrm{ex}}$ is of order $10 \mathrm{~nm}$. Here $\kappa=\left(K_{1} / \mu_{0} M_{s}^{2}\right)^{1 / 2}$ is the dimensionless hardness parameter and $l_{\mathrm{ex}}=\left(A / \mu_{0} M_{s}^{2}\right)^{1 / 2}$ is the exchange length which is about $2 \mathrm{~nm}$ for typical ferromagnets. ${ }^{9}$

(6) Magnetization reversal by coherent rotation. The critical coherence radius $R_{\mathrm{coh}}=\sqrt{ }(24) l_{\mathrm{ex}}{ }^{9}$
(7) An effective anisotropy constant $K_{\text {eff }}$ which may vary as $R^{-1}$ where $R$ is the particle radius.

A feature of magnetic fine particles is surface anisotropy. The effect was first discussed by Néel, ${ }^{10}$ who considered a phenomenological magnetoelastic expression and estimated the magnitude of the anisotropy as $K_{s} \approx 1 \mathrm{~mJ} \mathrm{~m}^{-2}$. This corresponds to an anisotropy energy per surface atom $\kappa_{s}=K_{s} \omega^{2 / 3}$ where $\omega$ is the atomic volume $(\approx 1.2$ $\times 10^{-29} \mathrm{~m}^{-3}$ for iron) equivalent to about $4 \mathrm{~K}$, which exceeds by an order of magnitude the values of bulk anisotropy or dipole-dipole interactions in $3 d$ magnets. In nanoparticles, with their large surface to volume ratio (Table I), surface anisotropy is expected to be second only to exchange in determining the magnetic properties.

Previous simulations of the magnetic ground state and hysteresis in ferromagnetic nanoparticles have been based on the Landau-Lifschitz-Gilbert equation ${ }^{11-13}$ using a RungeKutta algorithm. The elements are shrunk to the point where they represent only a few atoms, or even a single atom, ${ }^{14}$ and are treated as classical vectors. An alternative approach is to exploit the power of computer clusters to access the ground state and trace the field and time dependences of the spin configurations. We adopted the second approach in an earlier study of a ferromagnetic nanoparticles, where we were able to show that two types of spin structure develop in response to surface anisotropy. ${ }^{15}$ When the value of $\kappa_{s}$ is negligible in comparison to the exchange energy $J$, the particles adopt a collinear ferromagnetic configuration [Fig. 1(a)]. As $\kappa_{s}$ becomes increasingly positive, easy axes for the surface atoms 
TABLE I. Numbers of bulk and surface atoms for simple cubic particles.

\begin{tabular}{lcccccccc}
\hline \hline$\rho$ & $Z=3$ & $Z=4$ & $Z=5$ & $Z=6$ & $\langle Z\rangle$ & $N_{s}$ & $N$ & $N_{s} / N$ \\
\hline 2 & 24 & 0 & 0 & 8 & 3.75 & 24 & 32 & 0.75 \\
3 & 56 & 0 & 24 & 56 & 4.57 & 80 & 136 & 0.59 \\
4 & 72 & 24 & 48 & 136 & 4.89 & 144 & 280 & 0.51 \\
5 & 72 & 96 & 72 & 312 & 5.13 & 240 & 552 & 0.43 \\
6 & 120 & 96 & 120 & 576 & 5.26 & 336 & 912 & 0.37 \\
7 & 168 & 144 & 144 & 1016 & 5.36 & 456 & 1472 & 0.31 \\
8 & 248 & 120 & 264 & 1544 & 5.43 & 632 & 2176 & 0.29 \\
9 & 240 & 264 & 288 & 2320 & 5.51 & 792 & 3112 & 0.25 \\
10 & 320 & 288 & 360 & 3256 & 5.55 & 968 & 4224 & 0.23 \\
11 & 408 & 288 & 504 & 4416 & 5.59 & 1200 & 5616 & 0.21 \\
12 & 408 & 456 & 552 & 5792 & 5.63 & 1416 & 7208 & 0.20 \\
13 & 560 & 432 & 696 & 7640 & 5.65 & 1688 & 9328 & 0.18 \\
\hline \hline
\end{tabular}

which are normal to the surface cause the ferromagnetic configuration to deform into a "throttled" structure [Fig. 1(b)], also sometimes known as the "flower" structure. However, beyond a critical value $\kappa_{s} \approx J$, there is an abrupt transition to a "hedgehog" structure with no net moment [Fig. 1(c)].15,16 The singularity at the heart of the hedgehog is modified by dipole-dipole interactions. On the other hand, when $\kappa_{s}$ is negative, the easy directions lie parallel to the surface, and the spins adopt an "artichoke" configuration [Fig. 1(d)]. There are also reports of Monte Carlo simulations of twodimensional magnetic nanodots with surface anisotropy. ${ }^{17,18}$
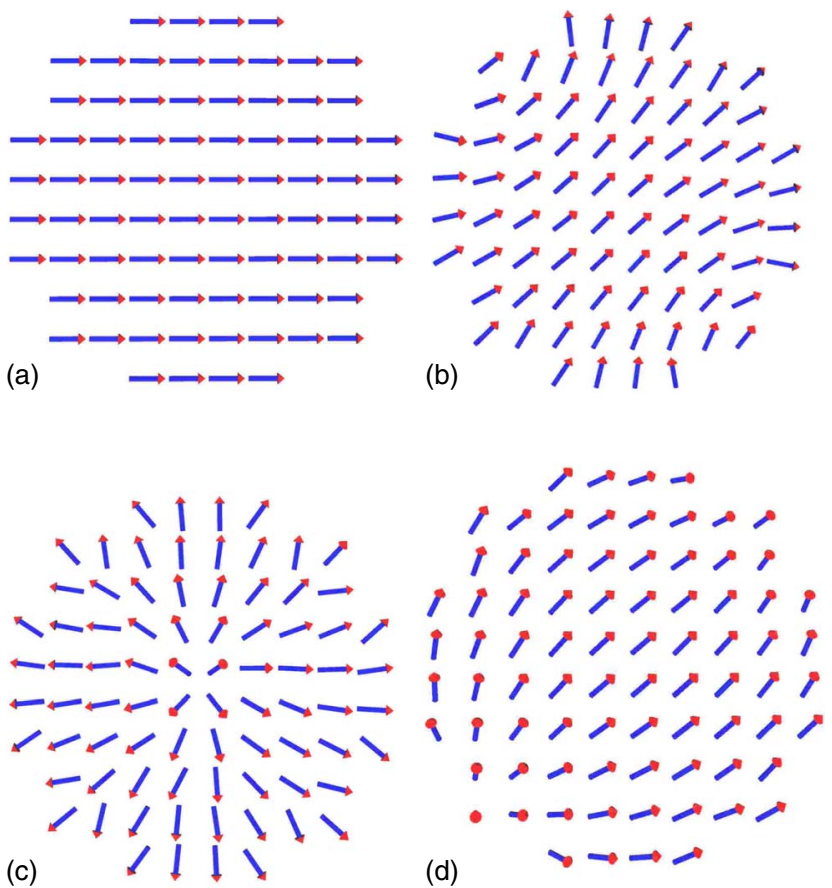

FIG. 1. (Color online) Spin configurations for a particle with a simple cubic structure and a radius $\rho=5$ (in units of interatomic spacing). (a) Collinear ferromagnetic configuration with surface anisotropy, $\kappa_{s}=0$; (b) throttled configuration; $\kappa_{s}=500$; (c) hedgehog configuration, $\kappa_{s}=2000$; and (d) artichoke configuration, $\kappa_{s}=$ -1500 .
Here the focus is on three-dimensional particles with positive $\kappa_{s}$. We investigate the magnetization processes and hysteresis for the throttled and hedgehog states. We examine the size dependence of the coercivity and consider the problem of evaluating the surface anisotropy from data on nanoparticle systems. Then we investigate the energy barriers between different spin states by simulating the magnetic relaxation. Finally, we review the magnitude of exchange, surface, and bulk anisotropy for a range of ferromagnetic materials, and suggest where to look for some of the predicted effects.

\section{METHODS}

Our model system is a spherical nanoparticle composed of atoms forming a simple cubic lattice with interatomic spacing $a$. The radius $R=\rho a$ is varied from $\rho=2$ to $\rho=13$, corresponding in practice to particles smaller than about $5 \mathrm{~nm}$. It is important to emphasize, as illustrated in Fig. 1, that the center of the spherical particle is not located on an atomic site, but at the centroid of the eight central atoms. Each magnetic site has $Z=6$ nearest neighbors in the volume and 3, 4, or 5 at the surface; the missing neighbors lead to the surface anisotropy. The sphere contains $N$ atoms with $N_{s}$ of them located on the surface. Values of $N$ and $N_{s}$, and the numbers of atoms with different coordinations are listed in Table I. A typical particle with $\rho=5$ is illustrated in Fig. 2 .

A vector spin $|\mathbf{S}|=1$ is associated with each atom. The system is described by a classical Heisenberg Hamiltonian, which includes terms denoting the nearest-neighbor exchange interaction, the anisotropy energy, the dipole interaction, and the Zeeman energy. For a given site $i$ it may be expressed as

$$
H_{i}=-\sum_{j=1}^{6} J \mathbf{S}_{i} \cdot \mathbf{S}_{j}-\kappa_{i}\left(\mathbf{S}_{i} \cdot \mathbf{n}_{i}\right)^{2}-\mu_{i}\left(\mathbf{B}_{\mathrm{dip}}+\mathbf{B}\right) \cdot \mathbf{S}_{i},
$$

where the sum is over the $j$ nearest neighbors. $J$ is the exchange coupling constant, $\mathbf{S}_{i}$ and $\mathbf{S}_{j}$ are the spins on sites $i$ and $j$, and $\kappa_{i}$ is the anisotropy constant, taken as $\kappa_{v}$ for all the sites with six nearest neighbors, or as $\kappa_{s}$ for the sites belonging to the surface. We take the bulk anisotropy $\kappa_{v}$ to be uniaxial along the $O z$ axis. The surface anisotropy direction is defined as 


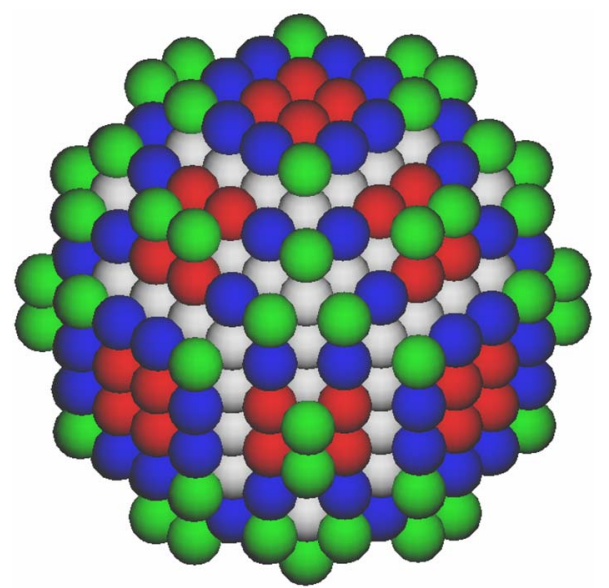

FIG. 2. (Color online) A particle with radius $\rho=5.43 \%$ of the atoms lies at the surface. Those with 3, 4, and 5 neighbors are colored green (light gray), dark blue (dark gray), red (medium light gray), and inner atoms are white.

$$
\boldsymbol{\sigma}_{i}=\Sigma_{j} \mathbf{r}_{i j} /\left|\Sigma_{j} \mathbf{r}_{i j}\right|
$$

These directions are $\langle 111\rangle$ for sites with $Z=3,\langle 110\rangle$ for $Z$ $=4$, and $\langle 100\rangle$ for $Z=5$. They lie close to the surface normal at every site. $\mathbf{B}_{\text {dip }}$ is the dipole field, $\mathbf{B}$ is the external applied field, and $\mu_{i}$ is the atomic magnetic moment in Bohr magnetons. For convenience in our simulation, we set $J=1000$ (ferromagnetic coupling) and choose $\kappa_{v}=0,10$, or 50 and $\kappa_{s}$ $=1-1200$. Having set the value of $J$, all the other interactions can be normalized to the exchange. When passing from the single-site Hamiltonian (1) to the Hamiltonian for the whole system, factors of $1 / 2$ appear in the exchange and dipole terms to avoid double counting. Noting that the maximum value of the dipole interaction for the particles studied does not exceed unity, we neglect this term in the following calculations.

The simulations have been performed in three steps: (i) First we analyze, as a function of particle size $\rho$, the spin structure and magnetization of the ground state, which is strongly dependent on surface anisotropy $\kappa_{s}$. (ii) The second step is to describe the initial magnetization curves and hysteresis loops for particles with a particular spin configuration and a chosen value of $\kappa_{s}$. (iii) Finally, the energy barriers separating hedgehog and throttled configurations are deduced from the Monte Carlo simulations, and analyzed as a function of the applied field.

All the simulations were performed on a Beowulf-class cluster composed of 35 PCs with dual Pentium III $600 \mathrm{MHz}$ processors. ${ }^{19}$ Starting from a random spin configuration at high temperature, the energy is minimized by simulated annealing using the Metropolis algorithm with a decreasing exponential law for temperature $T^{\gamma}$ with $\gamma=0.985$. We used unrestricted classical angular dynamics [the spin directions $(\theta, \phi)$ are chosen randomly]. The simulations were started at $T=2000$, well above $T_{C}$. The final temperature is normally $<1$. The number of Monte Carlo steps per spin was $10^{4}$. The time to reach the local equilibrium state for typical simulations was $1-100 \mathrm{~h}$, depending on particle size.

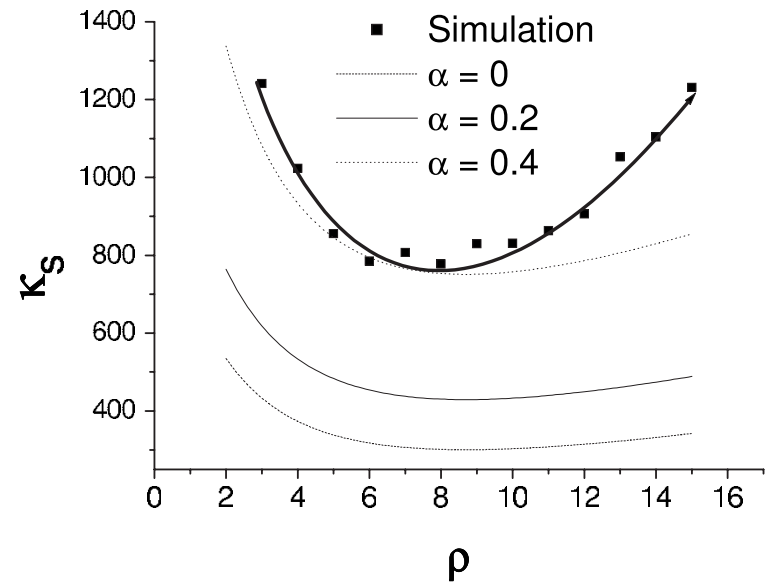

FIG. 3. Phase boundary between the hedgehog and throttled configurations when the exchange parameter $J=1000$ and $K_{v}=50$. Results of Monte Carlo simulations are indicated by the solid points. The lines are for an approximate analytical calculation described in the text. Error bars are smaller than the plot symbols.

For an extended simple cubic lattice the relation between exchange and Curie temperature $T_{C}$ is ${ }^{20}$

$$
T_{C}=1.44 J S^{2} .
$$

Hence $J=1000$ gives $T_{C}=1440$. This is approximately $T_{C}$ for Co.

\section{RESULTS}

\section{A. Ground state configuration}

We first determine the magnetic phase diagram as a function of particle radius $\rho$ with $N \approx 4 / 3 \pi \rho^{3}$ atoms in the particle and $N_{s} \approx 4 \pi \rho^{2}$ of them are lying on surface where they have surface anisotropy $\kappa_{s}$. The volume anisotropy $\kappa_{v}$ is varied $(0,10,50)$, while keeping the exchange coupling $J$ $=1000$. Energies of the hedgehog and throttled states are evaluated, and the lower energy state is the stable one. Energies calculated from the Monte Carlo simulation are compared to those of an approximate analytical calculation, described below. Results are presented in Fig. 3 .

The energy of the hedgehog configuration can be evaluated approximately, assuming that the magnetic moment $\mathbf{S}_{i}$ at site $i$ is given by $\mathbf{r}_{i} /\left|\mathbf{r}_{i}\right|$. Surface anisotropy energy $E_{s}$, volume anisotropy energy $E_{v}$, and exchange energy $E_{\mathrm{ex}}$ are given, respectively, by $-4 \pi \rho^{2} \kappa_{s},-4 \pi \rho^{2}(\rho / 3-1) \kappa_{v} / 3$, and $-\left(2 / 3 \pi \rho^{3}\langle Z\rangle J\right)\left\{1+3 \arctan (2 \rho) /\left(4 \rho^{3}\right)-3 /\left(2 \rho^{2}\right)\right\}$, where $\langle Z\rangle$ is the average number of nearest neighbors which is listed in Table I. The exchange term is obtained by considering the interaction of a pair of neighboring spins at a distance $\delta$ from the center of the particle, $J \cos \{2 \arctan [1 /(2 \delta)]\}$. Integrating over spherical shells which contain $4 \pi \delta^{2} d \delta$ such spins gives the expression for the exchange energy. As far as we know, no analytical expression exists for $\mathbf{S}_{i}$ in the throttled configuration, so we base an approximate analysis on a uniaxial ferromagnetic configuration [Fig. 1(a)]. In this case $\mathbf{S}_{i}=\mathbf{e}_{z}$, where $\mathbf{e}_{z}$ is the unit vector in the $z$ direction. The surface anisotropy, volume anisotropy, and exchange energies are 


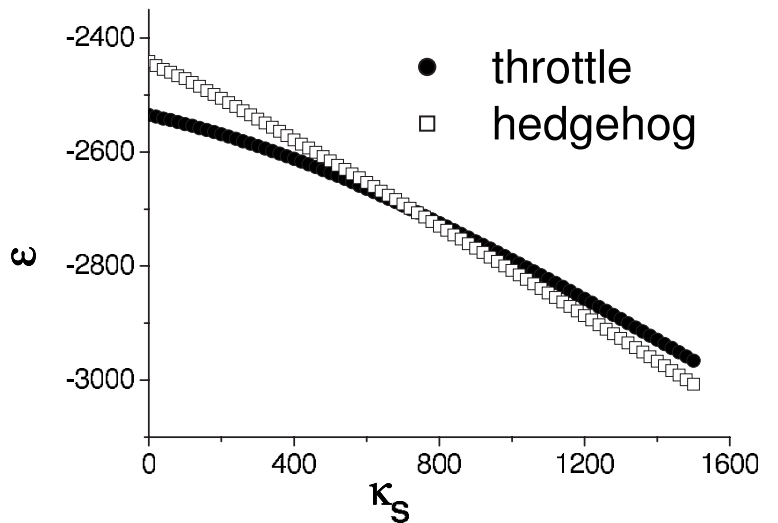

FIG. 4. Energy difference between the throttled and hedgehog states for a particle with radius $\rho=5$. Error bars are smaller than the plot symbols.

$-4 \pi^{2} \rho^{2} \kappa_{s} / 3,-4 \pi \rho^{2}(\rho / 3-1) \kappa_{v}$, and $-2 / 3 \pi \rho^{3}\langle Z\rangle J$, respectively. The phase boundary versus radius for given values of $\kappa_{v}$ and $J$ is obtained by equating the sum of these energies $E=E_{s}+E_{v}+E_{\mathrm{ex}}$ for each configuration. This leads to

$$
\kappa_{s}=\kappa_{v}(\rho / 3-1)-3\langle Z\rangle J / 4\left[\arctan (2 \rho) /\left(4 \rho^{2}\right)-1 /(2 \rho)\right] .
$$

To better approximate the throttled energy configuration, we need to reduce the surface anisotropy energy of the uniaxial configuration. This is done with a configuration which has a uniaxial ferromagnetic core and a surface with radially oriented spins. The expression for $\kappa_{s}$ then becomes

$$
\begin{aligned}
\kappa_{s}= & {\left[2(\rho / 3-1) \kappa_{v} / 3\right.} \\
& \left.-\langle Z\rangle J / 2\left\{\arctan (2 \rho) /\left(4 \rho^{2}\right)-1 /(2 \rho)\right\}\right] /(2 / 3-\alpha),
\end{aligned}
$$

with $\alpha$ ranging from 0 for the uniaxial configuration to $2 / 3$ for radial spins on the surface. The $\kappa_{s}(\rho)$ curves obtained from Eq. (5) with $\alpha=0.4$ are similar to those of the Monte Carlo simulations. The discrepancy between curves is certainly due to the approximation involved in assuming a quasiuniaxial configuration instead of the true throttled configuration.

The energy difference between the throttled and hedgehog states in the phase diagram of Fig. 3 is remarkably small. The dominant energy is the exchange, which is roughly $-\langle Z\rangle J / 2$ or $\approx 2500$ per site. Figure 4 shows the energy per site $\varepsilon=E / N$ as a function of $\kappa_{s}$ for the $\rho=5$ particle. The crossover occurs at $\kappa_{s} \approx 800$. To obtain the curves, $\kappa_{s}$ was varied in the course of the simulation. At $\kappa_{s}=0$ the energy difference is about 100 , or $4 \%$ of the exchange energy.

The hedgehog state is obviously twofold degenerate, in the sense that $\mathbf{S}_{i} \rightarrow-\mathbf{S}_{i}$ gives an inverted hedgehog with the same energy. In small particles, there is no continuous rotation symmetry on account of the stepped nature of the particle surface, which has the symmetry of a cube.

The throttled state is also twofold degenerate in the sense that $\mathbf{S}_{i} \rightarrow-\mathbf{S}_{i}$ gives a throttled state with the same energy. Since there is a net moment, there should be an easy axis. Our simulations, carried out in this case by cooling from temperature 2000 to 0.15 , show clearly the existence of $\langle 111\rangle$

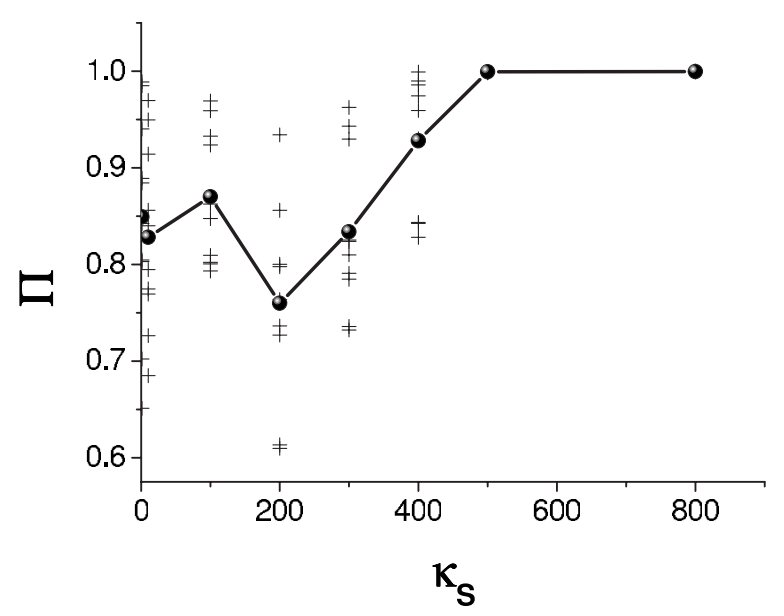

FIG. 5. Results of multiple Monte Carlo simulations to determine the easy axis of a particle with radius $\rho=5$. $\langle 111\rangle$ is easy when surface anisotropy $\kappa_{s}>450$. $\Pi$ is defined in the text.

easy axes when $\kappa_{s}>450$. A convenient way to represent the easy axis $\boldsymbol{\sigma}$ is to take the scalar product $\Pi$ of $\boldsymbol{\sigma}$ with $(1 / \sqrt{ } 3)[ \pm 1, \pm 1, \pm 1]$ in such a way that each component of the scalar product is positive. This gives $\Pi=1$ for $\langle 111\rangle, \Pi$ $=2 / \sqrt{ } 6(0.82)$ for $\langle 110\rangle$, and $\Pi=1 / \sqrt{ } 3(0.58)$ for $\langle 100\rangle$. From Fig. 5 it is evident that a $\langle 111\rangle$ direction is easy when $\kappa_{s}$ $>450$, but the orientation appears to be random for smaller values of $\kappa_{s}$. This is probably because the base temperature of the simulations is higher than the effective anisotropy per site $\kappa_{\text {eff }}$.

Easy axes arise wherever the surface anisotropy is able to deform the collinear ferromagnetic structure. The easy direction was also determined by fixing the direction of magnetization of a nucleus of eight atoms at the center of the particle and then carrying out a Monte Carlo simulation, cooling from $T=2000$, down to $T=0.15$. The energy is minimum for a $\langle 111\rangle$ direction (i.e., $\theta=54.7^{\circ}$ ), as shown in Fig. 6. The average anisotropy energy per site for the particle with $\rho$ $=5$ and $\kappa_{s}=500$ is only 1.4 .

Finally, we mention the case $\kappa_{s}<0$, which corresponds to easy-plane anisotropy of the surface sites. The effect of the

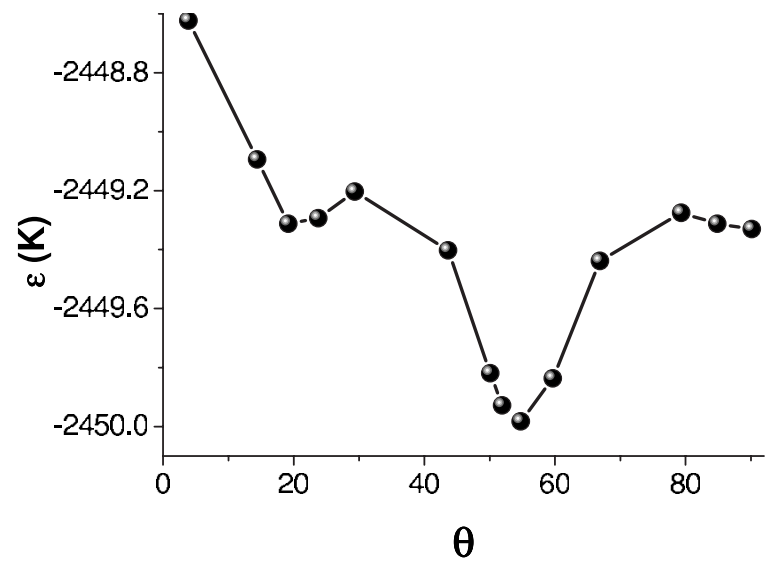

FIG. 6. Energy as a function of magnetization direction $\theta$ deduced by Monte Carlo simulations for a particle with radius $\rho=5$ and surface anisotropy $\kappa_{s}=500$. Error bars are smaller than the plot symbols. 

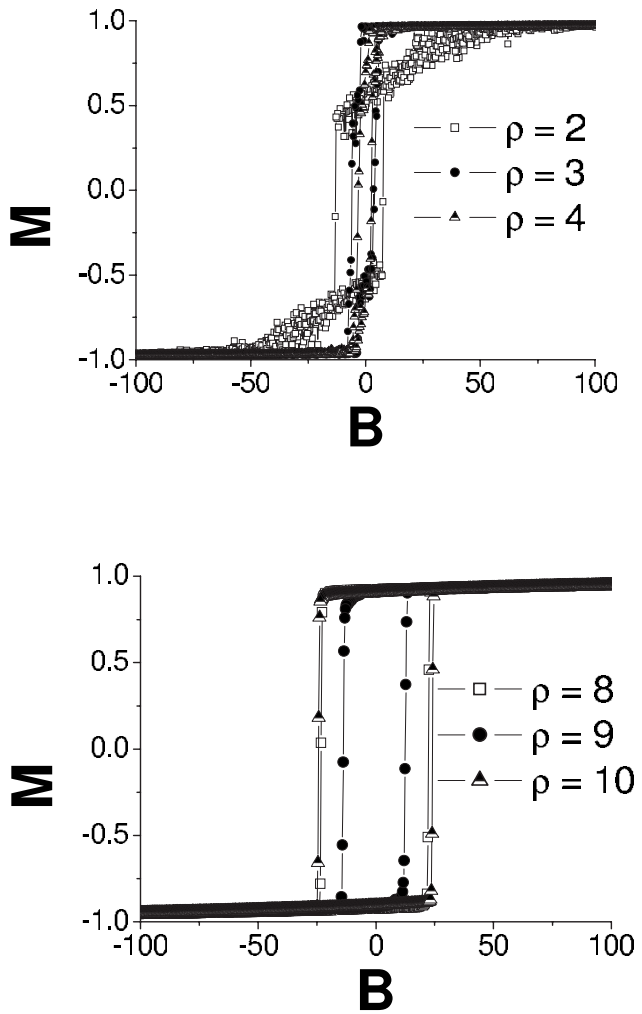
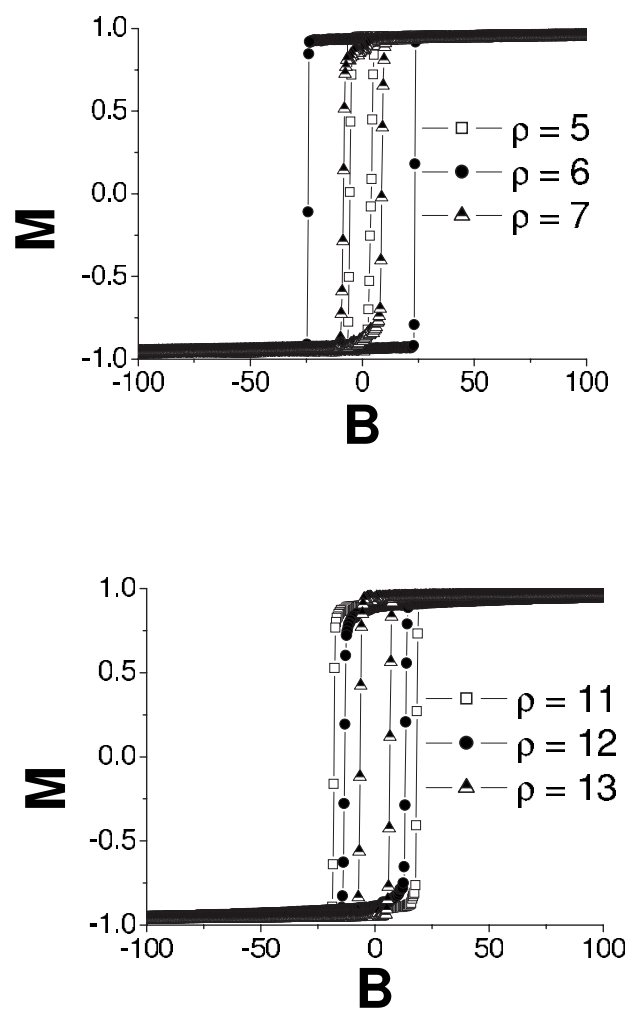

FIG. 7. Hysteresis loops simulated with the applied field along the [111] direction for particles with surface anisotropy $\kappa_{s}=400$ and different radii $\rho$. When error bars are not shown, they are smaller than the plot symbols. planar anisotropy is to induce an artichokelike spin structure, where the surface spins tend to lie parallel to the surface. Increasing the value of $\left|\kappa_{s}\right|$ leads to a continuous deformation of the ferromagnetic state. The easy axis is again $\langle 111\rangle$.

\section{B. Hysteresis loops}

Next we study the virgin magnetization curve and the complete hysteresis loop for particles with $J=1000$ and values of $\kappa_{s}$ which give either a throttled or a hedgehog configuration. For smaller values of $\kappa_{s}$ the rotation is coherent, and the nanoparticles behave like macrospins, as in the Stoner-Wohlfarth model. ${ }^{21-24}$ From the ground state, the magnetic field is increased in constant steps and the energy is minimized at each step using 80000 Monte Carlo iterations, after rejecting the first 10000 to allow for the approach to thermal equilibrium. The hysteresis is traced out by increasing the magnetic field $B$ from zero up to $B_{\max }\left(60<B_{\max }\right.$ $<500$ ), then reducing it to $-B_{\max }$ and again increasing $B_{\max }$ in order to describe a complete loop. The moment $\mu$ in Eq. (1) is taken as unity, so $B$ corresponds to the field in units of $k_{B} / \mu_{B} \approx 1.5 \mathrm{~T}$. In one case $\left(\rho=5, \kappa_{s}=1050, \kappa_{v}=10\right)$ this process was repeated ten times to be sure of the reproducibility of the results. In addition, minor loops have been investigated, where the maximum field is less than $B_{\max }$.

\section{Throttled configuration}

Here we choose $\kappa_{s}=400-800$ and $\kappa_{v}=0$, and apply the magnetic field along a [111] easy direction. There is therefore already a large moment in the virgin state. Particle radius is varied from $\rho=2$ to $\rho=13$. The hysteresis loops shown in Fig. 7 are for $\kappa_{s}=400$. The switching field $B_{s}$ varies irregularly and nonmonotonically with particle radius. The effective anisotropy constant deduced from the anisotropy field by the Stoner-Wohlfarth relation $B_{a}=2 \kappa_{\text {eff }} / \mu$ is plotted in Fig. 8. Again we take $\mu=1$.

An alternative method to evaluate the effective anisotropy is to apply a small magnetic field perpendicular to the easy direction, and to determine the slope of the initial magnetization curve (Fig. 9). The first step in the perpendicular magnetization curves corresponds to switching to the $\langle 111\rangle$ easy axis closest to the applied field direction. The second step corresponds to complete alignment with the applied field. Extrapolation of the initial curve to the spontaneous magnetization $m_{s}$ of the particle gives the anisotropy field $B_{a}$ which is also related to the effective anisotropy $\kappa_{\text {eff }}$ by $\kappa_{\text {eff }}$ $=B_{a} m_{s} / 2$. The two independent methods of determining $\kappa_{\mathrm{eff}}$

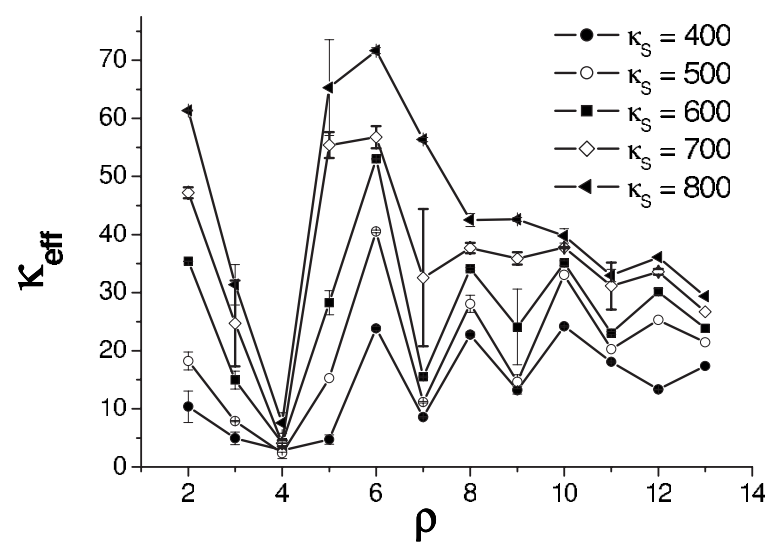

FIG. 8. Effective anisotropy $\kappa_{\text {eff }}$ deduced from the switching field in the hysteresis loops. Data are for different values of surface anisotropy $\kappa_{s}$. 


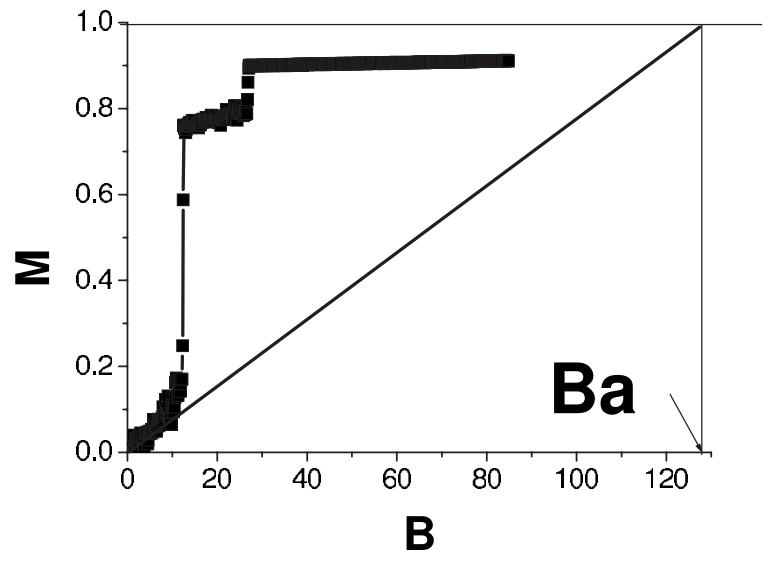

FIG. 9. Magnetization curve obtained when the field is applied perpendicular to the $\langle 111\rangle$ easy axis for a particle with radius $\rho=5$ and surface anisotropy $\kappa_{s}=400$ having a throttled spin configuration. Anisotropy field $B_{a}$ may be deduced from the initial slope, as described in the text.

are in rather good agreement, and the irregular variation with particle size in Fig. 8 is not an artifact. It is noteworthy that the values of $\kappa_{\text {eff }}$ are more than an order of magnitude less than $\kappa_{s}$ even though $20 \%-75 \%$ of the atoms in the particles with $2 \leq \rho \leq 12$ are surface atoms. This is because the aniso- tropy cannot be minimized at each surface atom site in the throttled magnetic structure.

\section{Hedgehog configuration}

Here we choose $\kappa_{s}=1100, \kappa_{v}=10$, and $\rho=5$. The field is applied along [001] and is again cycled from 0 to \pm 500 . In the virgin magnetization curve, three jumps marked a, b, and $\mathrm{c}$ in Fig. 10 occur before a final jump to join the demagnetization curve. These jumps each correspond to a shift in the center of symmetry of the magnetic moment distribution of one interatomic distance in the direction opposite to the field [Figs. 10(a)-10(d)]. It is interesting that this part of the magnetization curve is fully reversible, and the original hedgehog state can be recovered by reducing the field to zero. However, at a critical value of applied field (point d in Fig. 10), there is a jump to a throttled configuration, and the magnetization process becomes irreversible. On reducing $B$ to zero from point $\mathrm{e}$ in Fig. 10, the nanosphere remains in a throttled configuration, which is a metastable state in low fields, where the hedgehog energy is actually lower.

The virgin magnetization curve is reached again at point 1 in the first quadrant in Fig. 10, after magnetization reversal and increasing the magnetic field in the reverse direction. Remarkably, the displaced hedgehog configuration of Fig. $10(d)$ is found again, and on reducing $B$ to zero from point 1 a

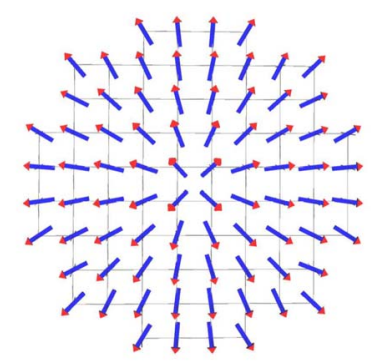

d

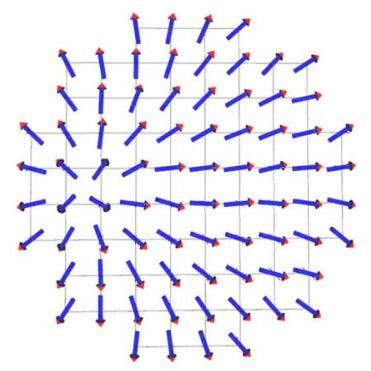

$\mathrm{g}$

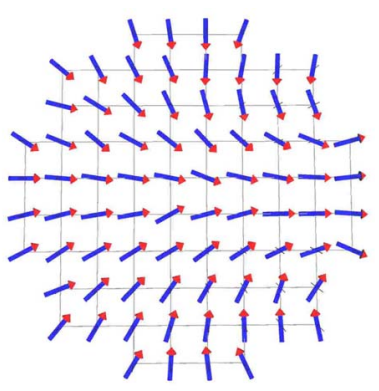

b

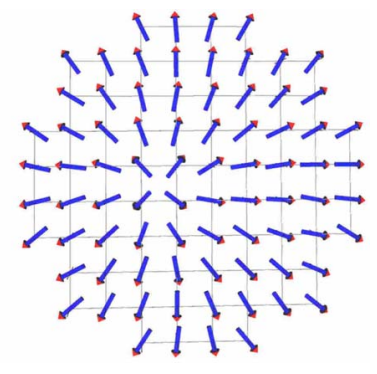

e

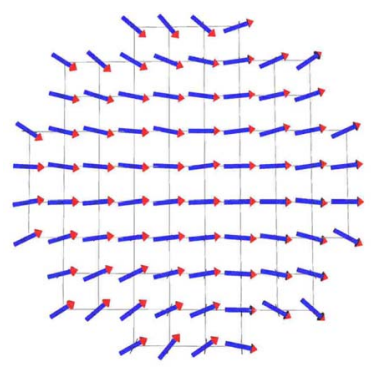

h

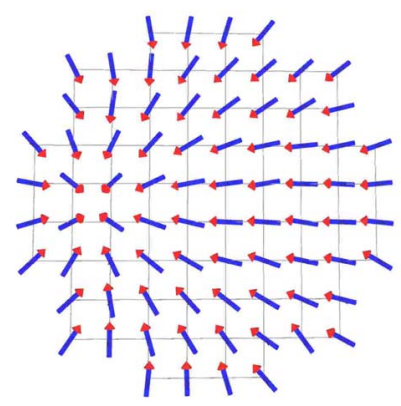

$\mathrm{c}$

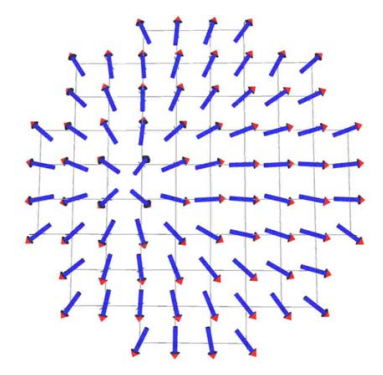

f

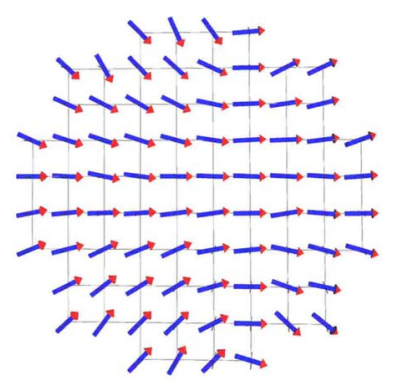

FIG. 10. (Color online) Hysteresis loop for a particle with radius $\rho=5$ and surface anisotropy $\kappa_{s}=1100$ originally in a throttled configuration. The magnetic configurations of the central plane are shown for the points labeled $\mathrm{a}-\mathrm{h}$ on the curve. 


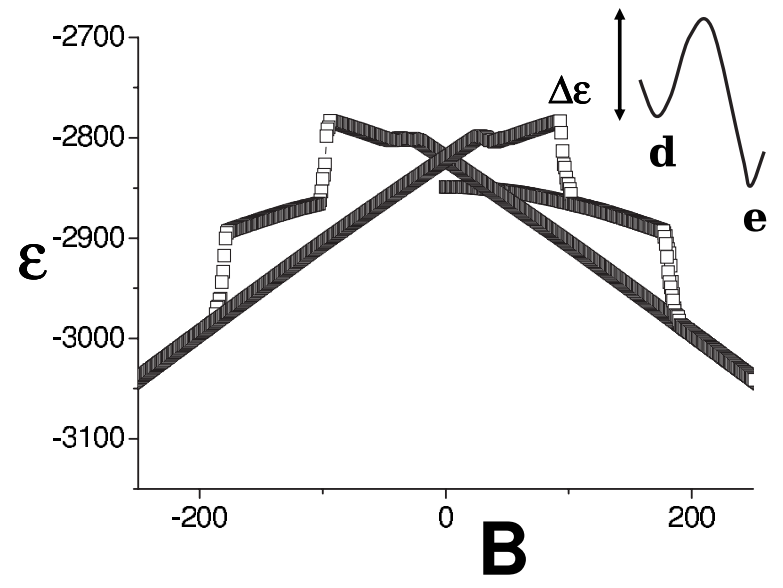

FIG. 11. Energies of the states traced out in the hysteresis loop in Fig. 10. The energy barrier for the transition $d \rightarrow e$ is also shown. Error bars are smaller than the plot symbols.

it is possible to recover the initial hedgehog state with zero magnetization. It is the nucleation of the group of four inward-pointing spins in Fig. 10(h) that decides the inwardpointing hedgehog. This recovery of reversible behavior after saturation is a most unusual effect of the strong surface anisotropy of these nanoparticles. It is only in such nanoparticles that the major loop can rejoin the initial curve.

\section{Energy barriers and magnetic relaxation}

Although the energies of the throttled and hedgehog configurations are very similar, the existence of broad hysteresis loops suggests that the barriers separating the two configurations are greater than their difference in energy. The energies of the two configurations involved in the hysteresis loop of Fig. 10 are shown in Fig. 11.

Thermal fluctuations of the magnetic moment of a singledomain ferromagnetic particle and its approach to equilibrium are well described by Néel-Brown model. ${ }^{24,25}$ The main assumptions in this model are that the magnetization is uniform and the anisotropy is uniaxial so that the relaxation can be described by a single relaxation time $\tau$. In the case of an isolated nanoparticle, the magnetic relaxation is given by an Arrhenius law of the form

$$
\tau=\tau_{0} \exp \left(\Delta E / k_{B} T\right)
$$

where $\tau_{0}$ is of order $10^{-9}-10^{-11} \mathrm{~s}$ and depends weakly on temperature, $\Delta E$ denotes the energy barrier separating the two configurations, $k_{B}$ is Boltzmann's constant, and $T$ is temperature. This model is widely used to describe the time dependence of magnetization of multiparticle assemblies where a logarithmic time dependence is found-for example, in $\gamma \mathrm{Fe}_{2} \mathrm{O}_{3},{ }^{26} \mathrm{Ni}$, Co, and Dy. ${ }^{27}$ This logarithmic time dependence arises from a broad distribution of relaxation times $\tau$ for the particles of different sizes.

Our objective here is to give a measure of the energy barrier $\Delta E$. Considering the relaxation from the hedgehog to the throttled configuration in a magnetic field close to that needed to make the jump $\mathrm{d} \rightarrow \mathrm{e}$ in Fig. 10, we performed 10000 runs in a fixed field in the range $80-185$ and at a fixed

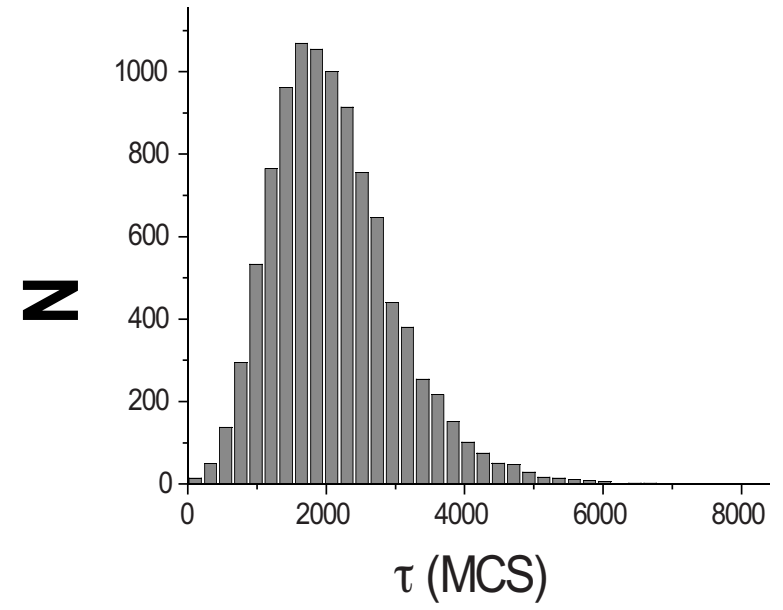

FIG. 12. Probability distribution for the switching time for a particle with radius $\rho=5$ surface anisotropy $\kappa_{S}=1100$, uniaxial volume anisotropy $\kappa_{v}=10$, applied field $B=185$, and $T=350 \mathrm{~K}$.

temperature in the range 400-650. For each run, the switching time $\tau$ for a single particle is recorded in units of Monte Carlo steps. Some typical data are shown in Fig. 12. They are fitted to give the average value $\langle\tau\rangle$. The slope of the plot of $\ln \langle\tau\rangle$ vs $1 / T$ gives the energy barrier $\Delta E$, and the variation of $\Delta E$ with field $B$ is shown in Fig. 13 for a particle with $\kappa_{s}$ $=1100, \kappa_{v}=10$, and $\rho=5$. The extrapolation of the energy barrier to zero field gives a barrier between the throttled and hedgehog configurations which is several times their energy difference. The result is reasonable and it justifies our observation that the Monte Carlo simulations find the local energy minima, which are a source of hysteresis.

When a sample contains particles with a distribution of particle size and shape, the abrupt variations seen in Fig. 8 are washed out. Assuming a log-normal distribution of size, the results for effective anisotropy as a function of average particle size shown in Fig. 14 are obtained.

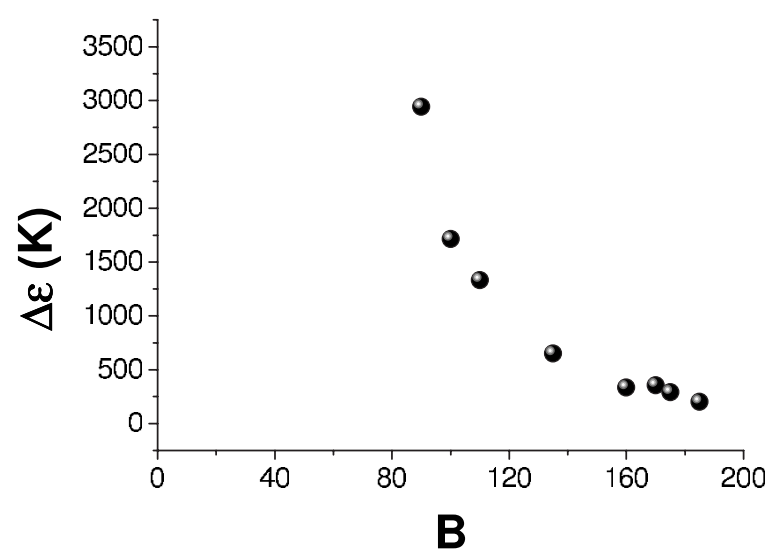

FIG. 13. Energy barrier for the transitions between the throttled and hedgehog configurations as function of applied field $B$. The particle has radius $\rho=5$, surface anisotropy $\kappa_{s}=1100$, and uniaxial volume anisotropy $\kappa_{v}=10$. 


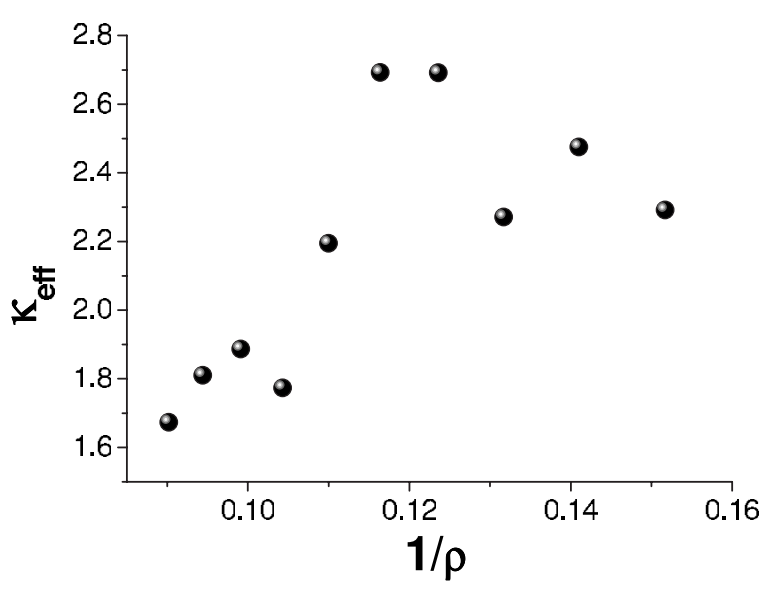

FIG. 14. Variation of $\kappa_{\text {eff }}$, the average anisotropy per site, for surface anisotropy $\kappa_{s}=500$, uniaxial volume anisotropy $\kappa_{v}=0$, and a log-normal distribution of particle size.

\section{DISCUSSION}

\section{A. Evaluation of surface anisotropy}

It is a common practice in the literature to evaluate the effective anisotropy constant of ferromagnetic thin films and nanoparticles from a formula such as

$$
K_{\mathrm{eff}}=K_{v}+\eta K_{s}
$$

where $\eta$ is an inverse length. For a film of thickness $t$ (two surfaces) $\eta=2 / t,{ }^{28}$ but for a nanosphere (following Bodker $e t$ $\left.a l .{ }^{2}\right)$ it is taken as $3 / R$, where $K_{\text {eff }}$ and $K_{v}$ have units $\mathrm{J} \mathrm{m}^{-3}$, whereas $K_{s}$ has units $\mathrm{J} \mathrm{m}^{-2}$. The basis of this formula is that the nanosphere contains $(4 / 3) \pi R^{3} / a^{3}$ atoms, $4 \pi R^{2} / a^{2}$ of which lie on the surface. If all the atoms contribute $\kappa_{v}$ $=K_{v} a^{3}$ to the anisotropy of the particle, and the surface atoms make an additional contribution of $\kappa_{s}=K_{s} a^{2}$, the total anisotropy is $(4 / 3) \pi(R / a)^{3} \kappa_{v}+4 \pi(R / a)^{2} \kappa_{s}$, and the effective anisotropy constant is therefore $\kappa_{\text {eff }}=\kappa_{v}+(3 / R) \kappa_{s}$.

This analysis is based on the implausible assumption that the anisotropy axes in the bulk and at the surface are all parallel. In fact, we have been supposing that the surface atoms have their easy directions roughly normal to the surface. In the limit of a large spherical particle with a uniform density of surface atoms and radial surface anisotropy $\kappa_{s}$ $\ll J$, so that the surface anisotropy does not modify the collinear ferromagnetic spin alignment, the contribution of $\kappa_{s}$ to $\kappa_{\text {eff }}$ is precisely zero. On account of their underlying crystal structure, small particles do not present a uniform density of surface atoms, as is illustrated in Fig. 2 for the particle with $\rho=5$. The anisotropy energy has been calculated for our simple cubic particles from $\rho=2$ up to $\rho=13$, and we conclude that $\langle 111\rangle$ are usually the easy axes, in agreement with the analysis of Garanin and Kachkachi, ${ }^{29}$ provided the surface anisotropy is sufficient to deform the collinear ferromagnetic structure. These authors found that $\langle 111\rangle$ axes are easy, regardless of the sign of the surface anisotropy in the Néel model.

From the plot of our size-averaged data for $\kappa_{s}=500$ as a function of $1 / \rho$ in Fig. 14, we find that the slope is not $3 \kappa_{s}$ $=1500$ but it is very much smaller. The procedure for deter-

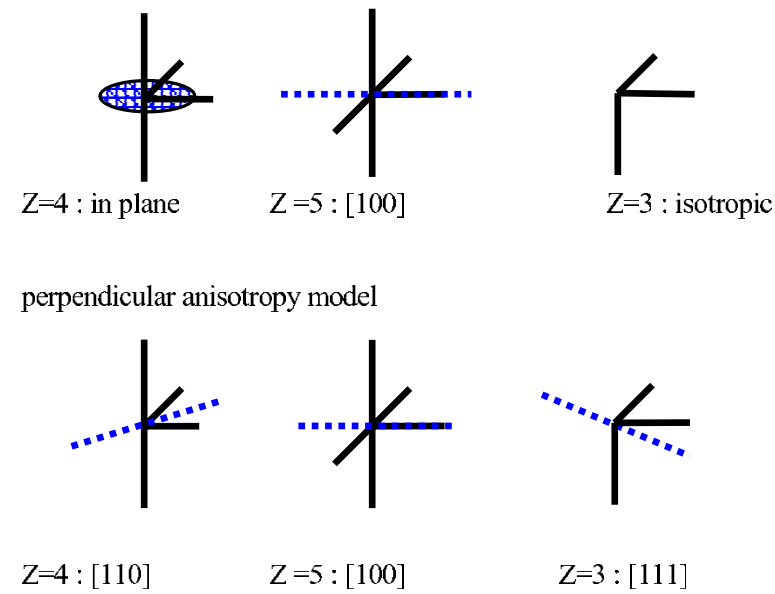

FIG. 15. (Color online) Comparison of the local easy directions for a simple cubic lattice in the Néel model (top row), and in the model used in this paper.

mining $\kappa_{s}$ from $\kappa_{\text {eff }}$ therefore underestimates its value by an order of magnitude. More serious is the absence of any compelling indication of a trend indicating a $1 / \rho$ variation. It is the specific surface structure of a nanoparticle that determines $\kappa_{\text {eff }}$, not simply its radius.

\section{B. Other models of surface anisotropy}

We have chosen an algorithm for defining surface anisotropy, which places the local anisotropy axes roughly normal to the surface and assumes easy-axis anisotropy. The effect of the algorithm is similar to radially directed anisotropy, transverse to the surface. ${ }^{30}$ The original Neel ${ }^{10}$ model was somewhat different. Néel assumed that the contribution of each nearest neighbor is $E=l / 2 \cos ^{2} \theta$ where $\theta$ is the angle between the direction of the local magnetization and the bond with the neighboring atom. His model gives the following results if $l$, which is the product of magnetostriction and elastic constants, is positive: for a surface perpendicular to $\langle 111\rangle$ there is no easy axis, for a surface perpendicular to $\langle 100\rangle$ the easy axis is $\langle 100\rangle$, and for a surface perpendicular to [011], [101], or [110] there is an easy plane perpendicular to $x, y$, and $z$, respectively, as shown in Fig. 15.

\section{Comparison with real ferromagnets}

The surface atoms have reduced symmetry where the axis of the electric field gradient (the leading crystal field term $A_{2}^{0}$ ) tends to lie perpendicular to the surface. In principle, this can lead to anisotropy which is perpendicular to the surface, or else lies in a plane parallel to the surface, depending on the sign of the crystal-field interaction. In the parallel case, other crystal-field terms such as $A_{2}^{2}$ will determine an easy direction in the plane. We therefore anticipate that among the rare earths, atoms with a positive quadrupole moment $(\mathrm{Sm}$, Er, Tm, and $\mathrm{Yb}$ ) will show perpendicular-to-surface anisotropy, and those with a negative quadrupole moment ( $\mathrm{Pr}, \mathrm{Nd}$, Tb, Dy, and Ho) will show a parallel-to-surface anisotropy. Among the $3 d$ atoms, similar arguments suggest that Co may show perpendicular anisotropy in a crystal field where the 
TABLE II. Typical values of physical parameters for selected ferromagnetic materials.

\begin{tabular}{lccccccc}
\hline \hline & $v^{\mathrm{a}}\left(\mathrm{nm}^{3}\right)$ & $T_{c}(\mathrm{~K})$ & $J(\mathrm{~K})$ & $K_{1}^{\mathrm{b}}\left(\mathrm{MJ} \mathrm{m}^{-3}\right)$ & $\kappa_{v}(\mathrm{~K})$ & $K_{s}\left(\mathrm{~mJ} \mathrm{~m}^{-2}\right)$ & $\kappa_{s}(\mathrm{~K})$ \\
\hline $\mathrm{Co}$ & 0.011 & 1390 & 959 & 0.5 & 0.4 & $\approx 1$ & 4 \\
$\mathrm{YCo}_{5}$ & 0.017 & 987 & 684 & 6.5 & 7.9 & $\sim 20$ & 95 \\
$\mathrm{FePt}$ & 0.028 & 750 & 520 & 6.6 & 13.4 & $\sim 34$ & 227 \\
$\mathrm{Er}$ & 0.031 & 85 & 57 & 7 & 15.4 & 14 & 100 \\
$\mathrm{~Tb}$ & 0.031 & 221 & 153 & -56 & -130 & -140 & -1000 \\
$\mathrm{TmNi}$ & 0.085 & 4.5 & 3 & 70 & 300 & 55 & 774 \\
$\mathrm{US}$ & 0.041 & 177 & 162 & 1000 & 3000 & 428 & 6000 \\
\hline \hline
\end{tabular}

${ }^{a}$ Volume per magnetic atom.

beading term in the anisotropy energy.

anisotropy of Fe is parallel, but another factor intervenes in the case of these metals, namely, the orbital moment associated with $3 d$ band electrons. Here the symmetry of the surface, which allows orbits to develop in the plane of the surface, but not in the perpendicular direction, suggests that the anisotropy due to spin-orbit coupling will inevitably be perpendicular to the surface. ${ }^{31}$ The magnetization of thin films indicates that surface anisotropy is usually perpendicular to the surface. ${ }^{32}$

It is important to relate the physical parameters $J, \kappa_{v}$, and $\kappa_{s}$ used in the Monte Carlo simulations to the values that characterize real materials. The numbers for a given simulation can all be scaled by a constant factor, without changing the magnetic ground state.

For the exchange, the bulk Curie temperature is used to determine $J$, using Eq. (3). With our definition of the Heisenberg Hamiltonian [Eq. (1), which differs by a factor of 2 from another common definition], Monte Carlo simulations with classical spins represented by $S=1$ give $k_{B} T_{C} / Z J$ $=0.24$. With $Z=6$, . Values of $J$ in Kelvin for a selection of ferromagnetic materials are listed in Table II.

For the bulk anisotropy, the measured uniaxial anisotropy constant $K_{1}$ (or $K_{1}^{c}$ for cubic materials) is converted to $\kappa_{v}$ in units of Kelvin by multiplying by the volume $v$ per magnetic ion, and dividing by Boltzmann's constant $k_{B}$.

The surface anisotropy is trickier to estimate. Evaluations based on Eq. (4) will tend to underestimate $\kappa_{s}$ for nanoparticles by as much as an order of magnitude for the reasons discussed in Sec. IV A. For cobalt, as an example, estimates from studies of nanoparticles have given values of $\kappa_{s}$ in the range $0.2-0.9 \mathrm{~mJ} \mathrm{~m}^{-3}, 5$ which correspond to $\kappa_{s}$ $=0.7-3.2 \mathrm{~K}$, whereas studies of ultrathin films give values of the surface anisotropy of order $0.7 \mathrm{~mJ} \mathrm{~m}^{-2}$ which corresponds to $\kappa_{s}=2.5 \mathrm{~K}^{33}$ Another approach is to take the bulk anisotropy in the most anisotropic cobalt-based alloys as a lower limit on the surface anisotropy. For example, CoPt and $\mathrm{YCo}_{5}$ have $K_{1}=4.9$ and $6.5 \mathrm{MJ} \mathrm{m}^{-3}$, respectively, which correspond to values of $\kappa_{s}$ of at least 9.8 or $7.9 \mathrm{~K}$. Much larger anisotropy, $\kappa_{s}=100 \mathrm{~K}$, is reported for a single cobalt atom on Pt. ${ }^{34}$ However, in any case, it is clear that cobalt and cobaltrich alloys with their high $T_{C}$ values lie far below the throttled and/or hedgehog boundary at $\kappa_{s} / J \approx 1$. The value of $\kappa_{s} / J$ is such that surface anisotropy will not significantly perturb the collinear ferromagnetic ground state. A similar expectation applies to FePt. Although $T_{C}$ is lower than it is for Co (Table II), the value of $J=520 \mathrm{~K}$ is still much greater than the bulk anisotropy $\kappa_{v}=13.4 \mathrm{~K}$, which is a lower limit on $\kappa_{s}$. Even if the ratio of surface to bulk anisotropy is similar to that in cobalt, we are in the region $\kappa_{s} / J \approx 0.5$ (Table II), ${ }^{35}$ where the ferromagnetic saturation is reduced by only about $10 \%$ by the throttled spin configuration.

The interesting effects we have found in our Monte Carlo simulations when $\kappa_{s} / J \approx 1$ are most likely to be manifest in compounds with strong surface anisotropy and weak exchange. The best candidates are rare-earth metals and alloys, and also some actinide-based ferromagnetic compounds. Erbium, for example, has a magnetic ordering temperature of $85 \mathrm{~K}$, which corresponds to $J=57 \mathrm{~K}$, and the anisotropy constant $K_{1}=7 \mathrm{MJ} \mathrm{m}^{-3}$ corresponds to $\kappa_{v}=15.4 \mathrm{~K}$. For the rare earths, $a b$ initio calculations ${ }^{36}$ suggest that the surface anisotropy is approximately twice as large as the bulk value; hence, for Er we expect that $\kappa_{s} \approx 100 \mathrm{~K}$. Erbium nanoparticles should therefore adopt a hedgehog state. Terbium, however, has $T_{C}=221 \mathrm{~K}$, which corresponds to $J=124 \mathrm{~K}$, and the hard-axis anisotropy constant $K_{1}=-56 \mathrm{MJ} \mathrm{m}^{-3}$ corresponds to $\kappa_{v}=-130 \mathrm{~K}$. The surface anisotropy expected is $\kappa_{s}=-1000 \mathrm{~K} .{ }^{36}$ Since the surface anisotropy is negative, and considerably larger than the exchange, it will lead to an artichoke configuration like that of Fig. 1(d).

More extreme examples with huge positive anisotropy are provided by $\mathrm{TmNi}_{5}$, where the exchange is weak, and by US, where the uranium anisotropy is exceptionally strong, as seen in Table II. A factor of 2 is used to estimate the surface anisotropy of the actinide, by analogy with the rare earths. Both these materials can be expected to exhibit hedgehog configurations.

\section{CONCLUSION}

Atomic-scale Monte Carlo simulations with classical spins indicate that significant modifications of the collinear ferromagnetic spin configuration can be expected in certain ferromagnetic nanoparticles due to surface anisotropy. There are interesting steps in the hysteresis loops, which resemble those found in larger nanoparticles, ${ }^{37,38}$ in molecular magnets, ${ }^{39,40}$ or in rare-earth alloy thin films, ${ }^{41}$ but which have a quite different physical explanation. In these small particles it is possible to associate the steps on the hysteresis loop with jumps between identifiable spin configurations. The reversibility of the steps in the first quadrant, after de- 
scribing an almost complete loop, is a signature of a hedgehog. These effects should be sought in nanoparticles of materials such as actinide or rare-earth compounds having $\kappa_{s} / J \approx 1$. We have suggested some examples.

There is no clear relation between particle radius and the effective surface anisotropy, but the approach of deducing $\kappa_{s}$ from Eq. (4) is inappropriate. It will tend to underestimate the surface anisotropy by an order of magnitude.

The commonly studied nanoparticles of the $3 d$ ferromagnetic elements and their alloys usually have $\kappa_{s} / J \ll 1$, and their spin configurations are barely modified by surface anisotropy. FePt is a case, however, where some measurable reduction in saturation magnetization due to a throttled spin configuration could be expected. The surface anisotropy in this case can also influence coercivity ${ }^{42}$ and precessional switching of magnetization. ${ }^{43}$

\section{ACKNOWLEDGMENTS}

This work was supported by the France-Ireland scientific exchange scheme, and by Science Foundation Ireland. The Monte Carlo simulation has been performed at "Lotus," a 70 processor, Beowulf-class, homemade parallel computer at LPEC (website: http://weblotus.univ-lemans.fr/w3lotus). We are grateful to Ralph Skomski for drawing to our attention the huge anisotropy of US.
${ }^{1}$ S. Sun, C. B. Murray, D. Weller, L. Folks, and A. Moser, Science 287, 1989 (2000).

${ }^{2}$ F. Bodker, S. Morup, and S. Linderoth, Phys. Rev. Lett. 72, 282 (1994)

${ }^{3}$ C. Chen, O. Kitakami, and Y. Shimada, J. Appl. Phys. 84, 2184 (1998).

${ }^{4}$ F. Gazeau, J. C. Bacri, F. Gendron, R. Perzynski, Y. L. Raikher, V. I. Stephanov, and E. Dubois, J. Magn. Magn. Mater. 186, 175 (1998).

${ }^{5}$ M. Respaud, J. M. Broto, H. Rakoto, A. R. Fert, L. Thomas, B. Barbara, M. Verelst, E. Snoeck, P. Lecante, A. Mosset, J. Osuna, T. Ould Ely, C. Amiens, and B. Chaudret, Phys. Rev. B 57, 2925 (1998).

${ }^{6}$ A. Punnoose, H. Magnone, M. S. Seehra, and J. Bonevich, Phys. Rev. B 64, 174420 (2001).

${ }^{7}$ E. De Biasi, C. A. Ramos, R. D. Zysler, and H. Romero, Phys. Rev. B 65, 144416 (2002).

${ }^{8}$ B. Barbara, Solid State Sci. 7, 668 (2005).

${ }^{9}$ L. Néel, Ann. Geophys. (C.N.R.S.) 5, 99 (1949).

${ }^{10}$ L. Néel, J. Phys. Radium 15, 255 (1954).

${ }^{11}$ D. A. Dimitrov and G. M. Wysin, Phys. Rev. B 50, 3077 (1994).

${ }^{12}$ D. A. Dimitrov and G. M. Wysin, Phys. Rev. B 51, 11947 (1995).

${ }^{13}$ H. Kachkachi and M. Dimian, Phys. Rev. B 66, 174419 (2002).

${ }^{14}$ M. Dimian and H. Kachkachi, J. Appl. Phys. 91, 7625 (2002).

${ }^{15}$ Y. Labaye, O. Crisan, L. Berger, J. M. Greneche, and J. M. D. Coey, J. Appl. Phys. 91, 8715 (2002).

${ }^{16}$ O. Iglesias and A. Labarta, J. Magn. Magn. Mater. 290-291, 738 (2005).

${ }^{17}$ V. E. Kireev and B. A. Ivanov, Phys. Rev. B 68, 104428 (2003).

${ }^{18}$ S. A. Leonel, I. A. Marques, P. Z. Coura, and B. V. Costa, J. Appl. Phys. 102, 104311 (2007).

${ }^{19}$ F. Calvayrac, Y. Labaye, and J. C. Gimel, Tech. Sci. Inform. 21, 1371 (2002).

${ }^{20}$ P. Peczak, A. M. Ferrenberg, and D. P. Landau, Phys. Rev. B 43, 6087 (1991).

${ }^{21}$ H. Kachkachi and E. Bonet, Phys. Rev. B 73, 224402 (2006).

${ }^{22}$ J. Mazo-Zuluaga, J. Restrepo, and J. Mejia-Lopez, Physica B 398, 187 (2007).

${ }^{23}$ H. Kachkachi and H. Mahboub, J. Magn. Magn. Mater. 316, 248 (2007).

${ }^{24}$ J. Restrepo, Y. Labaye, and J. M. Greneche, Physica B 384, 221 (2006).

${ }^{25}$ W. F. Brown, Phys. Rev. 130, 1677 (1963).
${ }^{26}$ R. Prozorov, Y. Yeshurun, T. Prozorov, and A. Gedanken, Phys. Rev. B 59, 6956 (1999).

${ }^{27}$ W. Wernsdorfer, E. Bonet Orozco, K. Hasselbach, A. Benoit, B. Barbara, N. Demoncy, A. Loiseau, H. Pascard, and D. Mailly, Phys. Rev. Lett. 78, 1791 (1997).

${ }^{28}$ H. J. G. Draaisma, W. J. M. de Jonge, and F. J. A. den Broeder, J. Magn. Magn. Mater. 66, 351 (1987).

${ }^{29}$ D. A. Garanin and H. Kachkachi, Phys. Rev. Lett. 90, 065504 (2003).

${ }^{30}$ H. Kachkachi and H. Mahboub, J. Magn. Magn. Mater. 278, 334 (2004).

${ }^{31}$ R. Skomski and J. M. D. Coey, Permanent Magnetism (Institute of Physics, Bristol, 1999).

${ }^{32} \mathrm{R}$ O'Handley, Magnetism and Magnetic Materials (Wiley, New York, 2001).

${ }^{33}$ D. Weller, J. Stöhr, R. Nakajima, A. Carl, M. G. Samant, C. Chappert, R. Mégy, P. Beauvillain, P. Veillet, and G. A. Held, Phys. Rev. Lett. 75, 3752 (1995).

${ }^{34}$ P. Gambardella, S. Rusponi, M. Veronese, S. S. Dhesi, C. Grazioli, A. Dallmeyer, I. Cabria, R. Zeller, P. H. Dederichs, K. Kern, C. Carbone, and H. Brune, Science 300, 1130 (2003).

${ }^{35}$ In our earlier paper, Ref. 14, we suggested that FePt nanoparticles may have a throttled spin structure, but there we omitted a factor 2 in the exchange, and were probably overgenerous in our estimate of $\kappa_{s}$.

${ }^{36}$ F. Welsch, M. Fähnle, and P. J. Jensen, J. Phys.: Condens. Matter 17, 2061 (2005).

${ }^{37}$ W. Wendorsfer, K. Hasselbach, D. Mailly, B. Barbara, A. Benoit, L. Thomas, and G. Suran, J. Magn. Magn. Mater. 145, 33 (1995).

${ }^{38}$ S. J. Hefferman, J. N. Chapman, and S. McVitie, J. Magn. Magn. Mater. 95, 76, (1991).

${ }^{39}$ Jonathan R. Friedman, M. P. Sarachik, J. Tejada, and R. Ziolo, Phys. Rev. Lett. 76, 3830 (1996).

${ }^{40}$ D. Gatteschi and R. Sessoli, J. Magn. Magn. Mater. 272-276, 1030 (2004).

${ }^{41}$ J. M. D. Coey, T. R. McGuire, and B. Tissier, Phys. Rev. B 24, 1261 (1981).

${ }^{42}$ W. Scholz, D. Suess, T. Schrefl, and J. Fidler, J. Appl. Phys. 95, 6807 (2004).

${ }^{43}$ M. Dimian and I. D. Mayergoyz, J. Appl. Phys. 97, $10 J 302$ (2005). 